\title{
Hintikka Style Game Rules for Semi-Fuzzy Quantifiers
}

\author{
Christian G. Fermüller \\ Theory and Logic Group 185.2 \\ Vienna University of Technology \\ 1040 Vienna, Austria \\ Email: chrisf@logic.at
}

\author{
Matthias F.J. Hofer \\ Theory and Logic Group 185.2 \\ Vienna University of Technology \\ 1040 Vienna, Austria \\ Email: hofer@logic.at
}

\begin{abstract}
Extending Hintikka's game for the evaluation of classical formulas, we explore the realm of quantifier rules that can be defined by combining several moves consisting of choices by the two strategic players, but also by a third non-strategic player 'Nature', representing random choices. The simple format of Hintikka-style games is compared to the seemingly much more general one of Giles's game for Lukasiewicz logic.
\end{abstract}

\section{INTRODUCTION}

One of the most important challenges in fuzzy logic is the design of adequate models of vague quantifier expressions like 'many', 'few', 'almost all', 'about half'. The literature on corresponding fuzzy quantifiers, initiated by Zadeh's [1], is almost insurmountable large; we refer to the monograph [2] and the more recent survey article [3] for an overview of relevant literature. Focusing on monadic (type $\langle 1\rangle$ ) quantifiers, a fuzzy quantifier is given by a truth function mapping any fuzzy set of domain objects $D$ into a truth value in $[0,1]$. Following a useful and well argued suggestion by Glöckner [2], such functions are to be determined in two separate steps: (1) define a semi-fuzzy quantifier, where the scope of the quantifier is a crisp (classical) predicate, and (2) lift the semi-fuzzy quantifier to a (fully) fuzzy quantifier in some systematic and uniform manner. Glöckner proposed an axiomatic approach for the second step and arrives at a corresponding quantifier fuzzification mechanism (QFM). Here we are mainly interested in the first step and moreover restrict attention to proportional quantifiers, like the ones in our opening sentence above, where the truth value of the quantified sentence only depends on the proportion of domain elements that satisfy the scope predicate of the quantifier. Even for this restricted (but important) class of semi-fuzzy quantifiers there are uncountably many different functions of type $[0,1] \rightarrow[0,1]$ that are prima facie reasonable candidates for the corresponding truth function of each quantifier of this type. It remains a challenge to single out particular (families of) truth functions in a systematic and principled manner, that respects criteria like linguistic adequateness, computational and conceptual simplicity, and compatibility with deductive

47th Intern. Symposium on Multiple-Valued Logic (ISMVL) (C)2017 IEEE DOI 10.1109/ISMVL.2017.57 fuzzy logics. The latter are systems of many-valued logics over the truth value set $[0,1]$; in particular the $t$-norm based logics at the core of mathematical fuzzy logic as presented, e.g., in the Handbook of Mathematical Fuzzy Logic [4].

The above mentioned challenge has been addressed in [5], [6] by extending Giles's game for Łukasiewicz logic [7]. This game models the stepwise reduction of logical complex assertions into atomic ones in a rule guided dialogue between two players. The players' payoff at a final state of the game is specified in terms of the total expected loss of money if each player bets for the success of dispersive experiments corresponding to atomic formulas. 'Dispersive' means that the (yes/no) results may differ when repeated; but a fixed success probability is associated with each experiment. In this manner Giles succeeded in deriving the truth functions of the logical connectives of Łukasiewicz logic from first principles about approximate reasoning, rather than just imposing these functions in an ad hoc fashion. While the game includes rules for standard existential and universal quantification, Giles did not consider (semi-)fuzzy quantifiers. The main ingredient of the semi-fuzzy quantifier rules presented in [5] and investigated more systematically in [6] are bets for and against certain numbers of instances of the scope formula that result from random choices of domain elements.

Better known than Giles's game, and arguably indeed more fundamental, is Hintikka's [8] characterization of truth in a model for classical first order formulas. It consists in a simple game with two players, one in the role of the proponent $\mathbf{P}$ and the other one in the role of the opponent $\mathbf{O}$, who stepwise reduce a given formula to one of its immediate subformulas until an atomic formula is reached. In this $\mathcal{H}$-game, as we shall call it, conjunction and universal quantification trigger choices by $\mathbf{O}$, while disjunction and existential quantification correspond to choices by $\mathbf{P}$; negation is expressed by a switch of roles between the players. At the final state of a run of the game $\mathbf{P}$ wins the game if the resulting atomic formula is true in the given interpretation, otherwise $\mathbf{O}$ wins.

While Giles's game ( $\mathcal{G}$-game) shares some central ideas with the $\mathcal{H}$-game, it features not only a more complex evaluation of final game states, but also a considerably more complex notion of a game state. Whereas a state of a $\mathcal{H}$-game is fully determined by a single formula and the current distribution of 
roles $(\mathbf{O} / \mathbf{P})$ between the two players, a state of the $\mathcal{G}$-game consists in two multisets of formulas, one for each player. Another type of generalization of Hintikka's game to fuzzy logics ( $\mathcal{E}$-games) is described in [9], which likewise features an additional component in the corresponding notion of a state, namely an explicit truth values attached to each formula in question. The corresponding game rules specify not only which subformula is selected for continuation, but also certain computations that are to be performed on the truth value in reducing compound formulas. We refer to [10] for a detailed presentation of these and some further variants of semantic games for fuzzy logics.

The aim of this paper is to explore the scope and limits of game rules for semi-fuzzy quantifiers that, in contrast to the $\mathcal{G}$-game and the $\mathcal{E}$-game, strictly adhere to 'Hintikka's principle': any state of the game is determined by a single (instance of a) subformula of the original formula and the information about which player acts as $\mathbf{P}$ and which as $\mathbf{O}$. In [11], the first author has investigated such Hintikka-style games for propositional fuzzy logics. Moreover the focus there is on quite simple, binary branching rules. Here we deal with quantification. Like in [5] and [6], random choices of domain elements are essential for obtaining interesting families of truth functions. However, note that Hintikka's principle prevents the reduction to simultaneous bets for and against whole multisets of corresponding instances of the quantified formula, that is central for Giles-style games. As we shall see, the expressive power of Hintikka-style games is nevertheless considerable if one considers rules that consist in several moves, combining random choices with those of the two strategic players.

\section{The BASIC $\mathcal{H}$-GAME}

Our starting point is Hintikka's classic semantic game [8], [12], called the basic $\mathcal{H}$-game here. Two players, called I (Myself) and You, can both act either as proponent $\mathbf{P}$ or as opponent $\mathbf{O}^{1}$ with respect to a formula $F$ built up using connectives $\wedge, \vee, \neg$ and the quantifiers $\forall, \exists$. Initially I act as $\mathbf{P}$ and You act as $\mathbf{O}$. My aim - or, more generally, P's aim at any state of the game - is to show that the initial formula is true in a given interpretation $\mathcal{M}$. The following rules refer to the outermost connective or quantifier of the current formula. For simplicity, we will assume that there is a constant for every element of the domain of $\mathcal{M}$.

$R_{\wedge}^{\mathcal{H}}$ : If the current formula is $F \wedge G$ then $\mathbf{O}$ chooses whether the game continues with $F$ or with $G$.

$R_{\vee}^{\mathcal{H}} \quad$ If the current formula is $F \vee G$ then $\mathbf{P}$ chooses whether the game continues with $F$ or with $G$.

$R_{\neg}^{\mathcal{H}}$ : If the current formula is $\neg F$, the game continues with $F$, except that the roles of the players are switched: the player who is currently acting as $\mathbf{P}$, acts as $\mathbf{O}$ at the next state, and vice versa for the current $\mathbf{O}$.

$R_{\forall}^{\mathcal{H}}:$ If the current formula is $\forall x F(x)$ then $\mathbf{O}$ chooses a constant $c$ and the game continues with $F(c)$.

\footnotetext{
${ }^{1}$ Hintikka uses Nature and Myself as names for the players and Verifier and Falsifer for the two roles. However, in our many-valued setting these names are problematic. Our terminology follows the handbook article [10].
}

$R_{\exists}^{\mathcal{H}}$ : If the current formula is $\exists x F(x)$ then $\mathbf{P}$ chooses a constant $c$ and the game continues with $F(c)$.

Except for $R_{\neg}^{\mathcal{H}}$, the players' roles remain unchanged. The game ends when the current formula is atomic. The player who is acting as $\mathbf{P}$ at the final state wins and the other player (acting as $\mathbf{O}$ ) loses, if this atomic formula is true in $\mathcal{M}$. We associate payoff 1 with winning and payoff 0 with losing. We also include the falsity constant $\perp$, signifying definite payoff 0 , among the atomic formulas. The game starting with formula $F$ is called the $\mathcal{H}$-game for $F$ under $\mathcal{M}$.

Theorem 1 (Hintikka). A formula $F$ is true in a (classical) interpretation $\mathcal{M}$ (also written as $v_{\mathcal{M}}(F)=1$ ) iff I have a winning strategy in the $\mathcal{H}$-game for $F$ under $\mathcal{M}$.

At the propositional level this result directly generalizes to Kleene-Zadeh logic $\mathrm{KZ}$, resulting from extending any given assignment of values in $[0,1]$ to atomic formulas as follows: given by the truth functions

$$
\begin{aligned}
& v_{\mathcal{M}}(F \wedge G)=\min \left(v_{\mathcal{M}}(F), v_{\mathcal{M}}(G)\right), \\
& v_{\mathcal{M}}(F \vee G)=\max \left(v_{\mathcal{M}}(F), v_{\mathcal{M}}(G)\right), \\
& v_{\mathcal{M}}(\neg F)=1-v_{\mathcal{M}}(F), \\
& v_{\mathcal{M}}(\perp)=0 .
\end{aligned}
$$

At the first order level we may define the semantics of the universal and the existential quantifier as follows, where we identify the elements of the domain $D$ with constants:

$$
\begin{aligned}
& v_{\mathcal{M}}(\forall x F(x))=\inf _{d \in D}\left(v_{\mathcal{M}}(F(d))\right), \\
& v_{\mathcal{M}}(\exists x F(x))=\sup _{d \in D}\left(v_{\mathcal{M}}(F(d))\right) .
\end{aligned}
$$

The $\mathcal{H}$-game rules remain unchanged here. Of course, such a straightforward lifting from classical to fuzzy logic (generalizing payoffs in $\{0,1\}$ to $[0,1]$ ) is only possible for $K Z$.

In the following we will restrict attention to finite domains and thus may assume that there are witnessing constants for all suprema and infima.

Definition 1. $w$ is called the value of the game for player $\boldsymbol{X}$ if $\boldsymbol{X}$ has a strategy that guarantees a payoff of at least $w$ for $\boldsymbol{X}$, while the opponent player has a strategy that ensures that $X$ 's payoff is at most $w$.

Theorem 2 ([6]). A formula $F$ evaluates to $v_{\mathcal{M}}(F)=w$ in a $\mathrm{KZ}$-interpretation $\mathcal{M}$ iff the $\mathcal{H}$-game for $F$ with payoffs matching $\mathcal{M}$ has value $w$ for Myself.

\section{General $\mathcal{H}$-game RUles - HintikKa's PRINCIPLE}

The $\mathcal{H}$-game does not provide rules for implication $(\rightarrow)$ or for equivalence $(\leftrightarrow)$. However such rules can easily be augmented by combining the basic building blocks — choices by $\mathbf{P}$, choices by $\mathbf{O}$, and role switch - within a single rule.

\footnotetext{
$R_{\rightarrow}^{\mathcal{H}}:$ If the current formula is $F \rightarrow G$ then $\mathbf{P}$ chooses whether (1) the game continues with $F$ or (2) with $G$; in the first case after a switch of roles.

$R_{\leftrightarrow}^{\mathcal{H}}$ : If the current formula is $F \leftrightarrow G$ then $\mathbf{O}$ chooses between one of the following two options:

(1) $\mathbf{P}$ gets to choose whether to continue with $F$ or
} 
with $G$; in the first case after a switch of roles.

(2) $\mathbf{P}$ gets to choose whether to continue with $F$ or with $G$; in the latter case after a switch of roles.

One could also consider connectives with more than two arguments. In particular, it is obvious how to formulate rules for $k$ ary conjunction or disjunction. In any case, the characteristic feature of the $\mathcal{H}$-game, which we want to maintain for all extensions of Hintikka's basic game throughout this paper, is the following.

Hintikka's Principle: The application of any game rule to the current formula $F$ results in a state that is fully determined by (an instance of) an immediate subformula of $F$ (or a truth constant) and a distribution of roles (I:P/You:O or I:O/You:P).

Obviously, only rules for connectives that are definable over $\{\vee, \wedge, \neg\}$ can be obtained in this manner. By functional completeness, for any truth function $f:\{0,1\}^{k} \rightarrow\{0,1\}$, there is a rule of the indicated type-combining choices by $\mathbf{P}$ and $\mathbf{O}$, projections to fixed subformulas or truth constants, and role switch, possibly in several rounds-for the corresponding classical connective. This observation generalizes to $K Z$, where the corresponding truth functions are those definable using $\min , \max , 1-x$, and projections to arguments or to the truth constant $\perp$.

Interesting options for generalizing the basic $\mathcal{H}$-game arise for quantifiers. Consider, e.g., the following rule:

$R_{E_{2}}^{\mathcal{H}}$ : If the current formula is $E_{2} x F(x)$ then $\mathbf{P}$ chooses two different constants $c_{1}$ and $c_{2}$ and, after that, $\mathbf{O}$ chooses whether the game continues with $F\left(c_{1}\right)$ or with $F\left(c_{2}\right)$.

It is straightforward to check that this rules characterizes the classical quantifier 'at least two'. As is well known, this quantifier can be defined from $\exists$ in the presence of identity. But the above rule enhances the expressive power of classical logic without the identity predicate. Again, it is straightforward to lift this observation to the many-valued setting.

\section{RANDOM CHOICES - INTRODUCING NATURE}

A quite powerful way to obtain game rules for connectives and quantifiers that are not expressible in $\mathrm{KZ}$ is to allow for uniformly random choices in addition to those by Myself and You. As is customary in game theory, such (uniformly) random choices can be understood as those of an additional, nonstrategic player, called Nature ( $\mathbf{N}$ for short). In particular it has been demonstrated in [11], that the expressivity of $\mathrm{KZ}$ is considerably extended already at the propositional level by introducing a new binary connective $\pi$ given by the following $\mathcal{H}$-game rule.

$R_{\pi}^{\mathcal{H}}$ : If the current formula is $F \pi G$ then $\mathbf{N}$ decides randomly whether the game continues with $F$ or $G$.

As shown in [11], the corresponding truth function is given by

$$
v_{\mathcal{M}}(F \pi G)=\frac{v_{\mathcal{M}}(F)+v_{\mathcal{M}}(G)}{2} .
$$

This observation can be lifted to specify the 'random choice quantifier' $\Pi$, introduced in [5] by the following rule.
$R_{\Pi}^{\mathcal{H}}: \quad$ If the current formula is $\Pi x F(x)$ then $\mathbf{N}$ (uniformly) randomly chooses a constant $c$ and the game continues with $F(c)$.

Remember that we assume that the domain $D$ of given interpretation $\mathcal{M}$ is always finite. Moreover we assume that constants and domain elements are in one-one correspondence. These assumptions lead to the following truth function for $\Pi$.

$$
v_{\mathcal{M}}(\Pi x F(x))=\operatorname{Prop}_{F}^{D}={ }_{d f} \frac{\sum_{i=1}^{|D|} v_{\mathcal{M}}\left(F\left(c_{i}\right)\right)}{|D|} .
$$

In the following we will suppress the reference to the domain $D$, since it will always be clear from the context. Also the reference to $F$ is usually clear, allowing us to simply write $p$ instead of Prop $_{F}$ when convenient. We are primarily interested in the case where $F$ is crisp, i.e., $\{0,1\}$-valued.

Notice that $R_{\pi}^{\mathcal{H}}$ nor $R_{\Pi}^{\mathcal{H}}$ are perfectly compatible with Hintikka's principle: there is still just a single next current formula and the role distribution $(\mathbf{P} / \mathbf{O}$ or $\mathbf{O} / \mathbf{P})$ for the two strategic players (I and You) remains unaffected by the nonstrategic player $\mathbf{N}$.

\section{A GENERAL FORMAT FOR QUANTIFIER RULES}

Combining Sections II, III, and IV we find all ingredients for a general format of quantifier rules for Hintikka-style games. We will only consider monadic (type $\langle 1\rangle$ ) quantifiers. As already indicated in Section III for the case of the connective $\leftrightarrow$, the key for obtaining game based definitions for a wider class of connectives and quantifiers is to compile several basic moves into a single rule. For monadic quantifiers this leads to the following scheme for rules referring to a current formula of the form $\mathrm{Q} x F(x)$ :

Rule Scheme for $\mathrm{Q} x F(x)$ :

Each move of the rule refers to some $\boldsymbol{X} \in\{\boldsymbol{O}, \boldsymbol{P}, \boldsymbol{N}\}$, where the player (in role) $\boldsymbol{X}$ may do just one of two things:

(A) choose a constant, or else

(B) choose among a finite number of given options for continuation; i.e., $\boldsymbol{X}$ chooses either the next move or else the particular instance $F(c)$ with which the rule ends, where $c$ is one of the previously chosen constants.

In addition, the rule may specify a role switch between the two strategic players (I and You) after the final move, i.e., before the game is continued. In any case, the finally chosen $F(c)$ is the current formula of the next round of the game.

Note that Hintikka's Principle is respected by our scheme. Every rule following the above scheme may be visualized as a tree of finite depth, where each node is associated with either $\mathbf{P}, \mathbf{O}$, or $\mathbf{N}$, and where the edges leaving the node correspond to the possible choices for that player in the corresponding move. Moves of type (A) induce a successor node for each domain element, whereas moves of type (B) induce a successor node for each of the given options for continuation. These options, according the scheme, may correspond to a further internal node (representing the next move) or else to a leave node (rule end), labeled by an instance of one of the scope formulas. Together with the possible indication of a role switch between 
$\mathbf{P}$ and $\mathbf{O}$, this next current formula determines the next state of the game.

In the next sections we will instantiate the above scheme in various ways and explore corresponding truth functions.

\section{EXAMPLES}

The following rules specify quantifiers where $\mathbf{O}$ may choose among a given finite number of constants, chosen by $\mathbf{N}$, for instantiating the scope formula.

$R_{\Pi_{k}^{\wedge}}^{\mathcal{H}}$ : If the current formula is $\Pi_{k}^{\wedge} x F(x)$, then $\mathbf{N}$ chooses $k$ (not necessarily different) constants $c_{1}, \ldots, c_{k}$, and then the player in role $\mathbf{O}$ chooses with which $F\left(c_{i}\right)$, where $1 \leq i \leq k$, to continue the game.

$R_{\bar{\Pi}_{k}^{\wedge}}^{\mathcal{H}}$ : Exactly as $R_{\Pi_{k}^{\wedge}}^{\mathcal{H}}$, except for a final role switch between Myself and You before the game is continued.

Note that these two families of quantifier rules indeed follow the general scheme of Section $\mathrm{V}$. They combine $k$ moves of type (A) by $\mathbf{N}$ with a single final move by $\mathbf{O}$, possibly followed by a role switch. Whereas the choices of the non-strategic player $\mathbf{N}$, by definition, are uniformly random; the choices of $\mathbf{O}$ are strategic and amount to conjunction. Remember that we focus on the case where the scope formula $F$ of a given quantifier occurrence is crisp. Together with our stipulation that constants and domain elements are in one-one correspondence this entails that $\operatorname{Prop}_{F}$ denotes the fraction of domain elements that satisfy the scope formula. These observations allow us to determine truth functions for our new quantifiers as follows.

Proposition 1. The rules $R_{\Pi_{k}^{\wedge}}^{\mathcal{H}}$ and $R_{\bar{\Pi}_{k}^{\wedge}}^{\mathcal{H}}$, applied to crisp scope formulas $F$, yield the following truth functions:

$$
\begin{aligned}
& v_{\mathcal{M}}\left(\Pi_{k}^{\wedge} x F(x)\right)=\left(\text { Prop }_{F}\right)^{k}, \\
& v_{\mathcal{M}}\left(\bar{\Pi}_{k}^{\wedge} x F(x)\right)=\left(1-\text { Prop }_{F}\right)^{k} .
\end{aligned}
$$

Proof. (1) First observe that the various component moves of the rule $R_{\Pi_{k}^{\wedge}}^{\mathcal{H}}$ indicate that $\Pi_{k}^{\wedge} x F(x)$ can be decomposed as follows in the context of the $\mathcal{H}$-game for logic $\mathrm{KZ}$, extended by rule $R_{\Pi}^{\mathcal{H}}$ of Section IV:

$$
v_{\mathcal{M}}\left(\Pi_{k}^{\wedge} x F(x)\right)=v_{\mathcal{M}}\left(\Pi x_{1} \ldots \Pi x_{k}\left(F\left(x_{1}\right) \wedge \cdots \wedge F\left(x_{k}\right)\right)\right)
$$

Since the random choices of the constants (N's choices) are by definition independent of each other, we obtain

$$
\frac{1}{|D|^{k}} \sum_{c^{1} \in D} \cdots \sum_{c^{k} \in D} \min \left(v_{\mathcal{M}}\left(F\left(c^{1}\right)\right), \ldots, v_{\mathcal{M}}\left(F\left(c^{k}\right)\right)\right)
$$

for $v_{\mathcal{M}}\left(\Pi_{k}^{\wedge} x F(x)\right)$. Since $F$ is a crisp formula, i.e. true (1) or false $(0)$ in each instance, we may rearrange the sums to get

$$
v_{\mathcal{M}}\left(\Pi_{k}^{\wedge} x F(x)\right)=\frac{\left(\sum_{i=1}^{|D|} v_{\mathcal{M}}\left(F\left(c_{i}\right)\right)\right)^{k}}{|D|^{k}}=\left(\text { Prop }_{F}\right)^{k} .
$$

(2) The second claim follows from the above considerations for the first one by taking into account that the final rule switch in $R_{\bar{\Pi}_{k}^{\mathcal{H}}}^{\wedge}$ (by Theorem 2) corresponds to negation and thus to the function $1-x$. From this observation we obtain the following in analogy to case (1):

$v_{\mathcal{M}}\left(\bar{\Pi}_{k}^{\wedge} x F(x)\right)=\frac{\left(\sum_{i=1}^{|D|}\left(1-v_{\mathcal{M}}\left(F\left(c_{i}\right)\right)\right)\right)^{k}}{|D|^{k}}=\left(1-\text { Prop }_{F}\right)^{k}$.

Corollary 1. For crisp scope formulas $F$ the following holds:

$$
v_{\mathcal{M}}\left(\bar{\Pi}_{k}^{\wedge} x F(x)\right)=v_{\mathcal{M}}\left(\Pi_{k}^{\wedge} x \neg F(x)\right) .
$$

In the proof of Proposition 1 we made use of the fact that the current formula of the form $\mathrm{Q} x F(x)$ corresponds to a compound formula using only the elementary random choice quantifier $\Pi$ and $\wedge$ and $\vee$. This observation can be generalized as expressed in the following proposition.

Proposition 2. Let $G$ be a formula built up from variants ${ }^{2}$ of a given formula $F(x)$ using the connectives $\wedge, \vee, \pi$, $\neg$ and the quantifiers $\forall, \exists$, and $\Pi$, such that all exhibited variable occurrences are bound. Then $G$ translates into a game rule instantiating the scheme presented in Section $V$ for the quantifier $\mathrm{Q}_{G}$ specified by $v_{\mathcal{M}}\left(\mathrm{Q}_{G} x F(x)\right)=v_{\mathcal{M}}(G)$.

Proof. (Sketch, relying on Theorems 1 and 2, as well as their generalizations to $\pi$ and $\Pi$ in [11] and [6], respectively.)

Occurrences of $\forall, \exists$, and $\Pi$ in $G$ correspond to moves of type (A) for $\mathbf{O}, \mathbf{P}$, and $\mathbf{N}$, respectively. Occurrences of $\wedge$, $\checkmark$, and $\pi$, correspond to moves of type (B) for $\mathbf{O}, \mathbf{P}$, and $\mathbf{N}$, respectively. Negation corresponds to role switch. Since the scheme only allows for role switch as a final step, it remains to observe that all occurrences of negation signs $(\neg)$ in $G$ can be pushed in front of occurrences of atomic formulas. This is clear for conjunctions, disjunctions, and universal and existential quantification, since the classical equivalences, $v_{\mathcal{M}}(\neg(A \wedge B))=v_{\mathcal{M}}(\neg A \vee \neg B), v_{\mathcal{M}}(\neg(A \vee B))=$ $v_{\mathcal{M}}(\neg A \wedge \neg B), \quad v_{\mathcal{M}}(\neg \forall x A(x))=v_{\mathcal{M}}(\exists x \neg A(x))$, and $v_{\mathcal{M}}(\neg \exists x A(x))=v_{\mathcal{M}}(\forall x \neg A(x))$ hold for $\mathrm{KZ}$ as well. But we also have $v_{\mathcal{M}}(\neg \Pi x A(x))=v_{\mathcal{M}}(\Pi x \neg A(x))$ as well as $v_{\mathcal{M}}(\neg(A \pi B))=v_{\mathcal{M}}(\neg A \pi \neg B)$, which completes the proof.

We illustrate Proposition 2 by extracting a rule from $G=$ $\Pi x(\neg F(x) \vee \forall y(F(y) \pi \exists z \neg F(z)))$. Following the above proof sketch we obtain:

$R_{Q_{G}}^{\mathcal{H}}:$ If the current formula is $\mathrm{Q}_{G} x F(x)$, then

1 [type (A) move for $\mathbf{N}$ ]: $\mathbf{N}$ chooses a constant $c$.

2 [type (B) move for $\mathbf{P}$ ]: $\mathbf{P}$ chooses between 3 and 4 .

3 [end]: The game continues with $F(c)$ after role switch.

4 [type (A) move for $\mathbf{O}$ ]: $\mathbf{O}$ chooses a constant $d$.

5 [type (B) move for $\mathbf{N}$ ]: $\mathbf{N}$ chooses between 6 and 7.

6 [end]: The game continues with $F(d)$ (no role switch).

7 [type (A) move for $\mathbf{P}$ ]: $\mathbf{N}$ chooses a constant $e$.

8 [end]: The game continues with $F(e)$ after role switch.

\footnotetext{
${ }^{2} \mathrm{~A}$ variant of $F(x)$ results from uniformly renaming all free occurrences of the exhibited variable $x$ in $F$. In particular $F(x)$ and $F(y)$ denote variants of each other.
} 
As we have seen, the truth functions of $\Pi_{k}^{\wedge}$ and $\bar{\Pi}_{k}^{\wedge}$ depend on the proportion $\operatorname{Prop}_{F}$ of domain elements satisfying the crisp scope predicate $F$ in a very straightforward manner. Next we show how more involved game rules allow us to match more complex truth functions that reflect binomially distributed expected payoff in the corresponding game.

\section{Binomial Distribution}

We start by synthesizing a quantifier rule induced by

$$
G=\Pi x((F(x) \wedge \Pi y \neg F(y)) \vee(\neg F(x) \wedge \Pi y F(y))) .
$$

Writing $\Pi_{1}^{1}$ for $Q_{G}$, we obtain the following rule according to the proof sketch for Proposition 2:

$R_{\Pi_{1}^{1}}^{\mathcal{H}}: \quad$ If the current formula is $\Pi_{1}^{1} x F(x)$, then

1: $\mathbf{N}$ chooses a constant $c$.

2: $\mathbf{P}$ chooses between 3 and 3'.

3: $\mathbf{O}$ chooses between the following options: - to continue the game with $F(c)$, or - to go to move 4.

4: $\mathbf{N}$ chooses a constant $d$.

5: The game continues with $F(d)$ after role switch. 3'-5': Like 3-5, except for a role switch before continuation with $F(c)$, instead of before continuation with $F(d)$.

To see that this rule is indeed adequate relative to $G$, note that the choices of a constant by $\mathbf{N}$ in moves 1,4 , and 4' of $R_{\Pi_{1}^{1}}^{\mathcal{H}}$ correspond to occurrences of $\Pi$ in $G$ (cf. Section IV). By Hintikka's Theorem 1, lifted to the many-value setting in Theorem 2, P's choice between the two options in move 2 corresponds to a disjunction. Similarly, the choices by $\mathbf{O}$ in moves 3 and 3' of $R_{\Pi_{1}^{1}}^{\mathcal{H}}$ correspond to conjunctions.

The truth function for $\Pi_{1}^{1}$, understood as semi-fuzzy quantifier, is as follows.

Lemma 1. For crisp scope formulas $F$ we have

$$
v_{\mathcal{M}}\left(\Pi_{1}^{1} x F(x)\right)=2 \operatorname{Prop}_{F}\left(1-\operatorname{Prop}_{F}\right) .
$$

Proof. Let $c$ be the constant chosen by $\mathbf{N}$ in move 1 of $R_{\Pi_{1}^{1}}^{\mathcal{H}}$. Since $F$ is crisp, we may distinguish two cases:

(1) $v_{\mathcal{M}}(F(c))=1$ : in this case $\mathbf{P}$ will choose option 3 in move 2 of rule $R_{\Pi_{1}^{1}}^{\mathcal{H}}$ to increase her expected payoff. This corresponds to picking the left disjunct of the formula in the scope of $\Pi x$ in $G$. (In fact the choice is irrelevant if $|D|=$ 1. However if $|D|>1$, the expected value for $\mathbf{P}$ is indeed higher for this option leading to the left disjunct of $G$.) Next $\mathbf{O}$ (in move 3) will rationally choose to continue with move 4, corresponding to the focus on the subformula $\Pi y \neg F(y)$ of $G$. The expected value for $\mathbf{P}$ in this case therefore is $1-$ Prop $_{F}$. (2) $v_{\mathcal{M}}(F(c))=0$ : in this case $\mathbf{P}$ rationally will choose option 3 ' in move 2. This corresponds to picking the right disjunct of $G$. Again $\mathbf{O}$ (in move 3) will choose to continue with 4, which amounts to the focus on the subformula $\Pi y F(y)$. The expected value for $\mathbf{P}$ in this case therefore is $\operatorname{Prop}_{F}$.

Since case (1) occurs with probability $\operatorname{Prop}_{F}$ and case (2) with probability $1-$ Prop $_{F}$ we obtain

$\operatorname{Prop}_{F}\left(1-\operatorname{Prop}_{F}\right)+\left(1-\operatorname{Prop}_{F}\right) \operatorname{Prop}_{F}=2 \operatorname{Prop}_{F}\left(1-\operatorname{Prop}_{F}\right)$ for the overall expected value.

In the above proof, although not strictly necessary, we have appealed to the decomposition of $\Pi_{1}^{1} x F(x)$ into a formula involving only $\Pi, \wedge, \vee$, and $\neg$. We will now go into the inverse direction and first determine truth functions of quantifiers induced by such formulas and then conclude from Proposition 2 that corresponding $\mathcal{H}$-game rules can be synthesized.

For the following recursive definition recall the quantifiers $\Pi_{k}^{\wedge}$ from Section VI and note that $\Pi_{1}^{\wedge} x F(x)=\Pi x F(x)$.

Definition 2. The quantifiers $\Pi_{m}^{k}$ are given by

$$
\begin{gathered}
\Pi_{0}^{k} x F(x)=\Pi_{k}^{\wedge} x F(x), \quad \Pi_{m}^{0} x F(x)=\bar{\Pi}_{m}^{\wedge} x F(x), \quad \text { and } \\
\Pi_{m}^{k} x F(x)=\Pi x\left(\left(F(x) \wedge \Pi_{m}^{k-1} y \neg F(y)\right) \vee\left(\neg F(x) \wedge \Pi_{m-1}^{k} y F(y)\right) .\right.
\end{gathered}
$$

Theorem 3. Applied to crisp scope formulas $F$ the truth functions for $\Pi_{m}^{k}$ are as follows:

$$
v_{\mathcal{M}}\left(\Pi_{m}^{k} x F(x)\right)=\left(\begin{array}{c}
k+m \\
k
\end{array}\right)\left(\operatorname{Prop}_{F}\right)^{k}\left(1-\operatorname{Prop}_{F}\right)^{m} .
$$

Proof. The two base cases follow from Proposition 1. For the case $k=1$ we argue like in the special case of $\Pi_{1}^{1}$ in the proof of Proposition 1. Writing $v_{m}^{k}$ for $v_{\mathcal{M}}\left(\Pi_{m}^{k} x F(x)\right), \bar{v}_{m}^{k}$ for $v_{\mathcal{M}}\left(\Pi_{m}^{k} x \neg F(x)\right)$ and $p$ for $\operatorname{Prop}_{F}$ we obtain

$$
v_{m+1}^{1}=p \bar{v}_{m+1}^{0}+(1-p) v_{m}^{1}
$$

and hence by Proposition 1 and induction on $m$

$$
\begin{aligned}
v_{m+1}^{1} & =p(1-p)^{m+1}+(1-p)\left(\begin{array}{c}
m+1 \\
1
\end{array}\right) p(1-p)^{m} \\
& =(m+1+1) p(1-p)^{m+1}=\left(\begin{array}{c}
m+2 \\
1
\end{array}\right) p(1-p)^{m+1} .
\end{aligned}
$$

The case for $m=1$ is analogous. Generally, for $m>1$,

$$
v_{m}^{k+1}=p \bar{v}_{m}^{k}+(1-p) v_{m-1}^{k+1}
$$

and therefore, by induction,

$$
\begin{aligned}
v_{m}^{k+1}= & p\left(\begin{array}{c}
k+m \\
k
\end{array}\right) p^{k}(1-p)^{m}+ \\
& (1-p)\left(\begin{array}{c}
m+1+m-1 \\
k+1
\end{array}\right) p^{k+1}(1-p)^{m-1} \\
= & \left(\begin{array}{c}
k+m+1 \\
k+1
\end{array}\right) p^{k+1}(1-p)^{m} .
\end{aligned}
$$

The case for increasing $k$ while fixing $m$ is analogous.

From Theorem 3 and Proposition 2 we obtain:

Corollary 2. For every $k, m \geq 0$ a game rule instantiating the general scheme of Section $V$ can be defined for the semi-fuzzy quantifier with the truth function

$$
v_{\mathcal{M}}\left(\Pi_{m}^{k} x F(x)\right)=\left(\begin{array}{c}
k+m \\
k
\end{array}\right)\left(\operatorname{Prop}_{F}\right)^{k}\left(1-\operatorname{Prop}_{F}\right)^{m} .
$$




\section{COMPARISON WITH $\mathcal{G}$-GAME BASED RULES}

As mentioned in the Introduction, game rules for semifuzzy quantifiers based on Giles's game ( $\mathcal{G}$-game) [7] for Łukasiewicz logic $Ł$ have been presented in [5] and in [6]. These rules refer to a much richer notion of a game state, given by

$$
\left[F_{1}, \ldots, F_{m} \mid G_{1}, \ldots, G_{n}\right] \text {, }
$$

where $\left\{F_{1}, \ldots, F_{m}\right\}$ is the multiset of Ł-formulas currently asserted by You, called your tenet, and $\left\{G_{1}, \ldots, G_{n}\right\}$ is the multiset of augmented formulas currently asserted by Myself, called my tenet. At any non-final state a current (non-atomic) formula $F$ gets picked. If this formula occurrence $F$ is in my tenet, then $\mathbf{I}$ act as $\mathbf{P}$ and you as $\mathbf{O}$ for $F$, and vice versa if $F$ is in your tenet. At the final state, where all occurring formulas are atomic, the payoff for Myself is given by

$$
m-n+1+\sum_{1 \leq i \leq n} v_{\mathcal{M}}\left(G_{i}\right)-\sum_{1 \leq i \leq m} v_{\mathcal{M}}\left(F_{i}\right) .
$$

We may view the $\mathcal{H}$-game as a very restricted version of the $\mathcal{G}$-game, where each state is either of the form $[\mid F]$ signaling the role distribution I:P/You:O or of the form $[F \mid]$, signaling the role distribution I:O/You:P. Indeed, the $\mathcal{G}$-game rules for (weak) conjunction $(\wedge)$, disjunction $(\vee)$, and for the universal and existential quantifiers $(\forall$ and $\exists$ ) directly correspond to the rules $R_{\wedge}^{\mathcal{H}}, R_{\vee}^{\mathcal{H}}, R_{\forall}^{\mathcal{H}}$, and $R_{\exists}^{\mathcal{H}}$ reviewed in Section II.

The $\mathcal{G}$-game setting admits the following rule [5]:

$R_{\Pi_{m}^{k}}^{\mathcal{H}}$ : If the current formula is $\Pi_{m}^{k} x F(x)$, then $k+m$ (not necessarily different) constants are chosen randomly. $\mathbf{P}$ picks $k$ of those constants, say $c_{1}, \ldots, c_{k}$ and bets for $F\left(c_{1}\right), \ldots, F\left(c_{k}\right)$, while simultaneously betting against $F\left(c_{1}^{\prime}\right), \ldots, F\left(c_{m}^{\prime}\right)$, where $c_{1}^{\prime}, \ldots, c_{m}^{\prime}$ are the remaining random constants.

Betting for a formula $F$ in the $\mathcal{G}$-game means that $F$ is added to P's tenet, while betting against $F$ means that $F$ is added to O's tenet, while an occurrence of $\perp$ is added to P's tenet. Moreover a principle of limited liability is in place. In this case this means that, after the randomly chosen constants are revealed, $\mathbf{P}$ may decide to replace the current formula by an occurrence of $\perp$ instead of proceeding as specified in $R_{\Pi^{k}}^{\mathcal{H}}$.

It is no accident that we have used the same symbol $\left(\Pi_{m}^{k}\right)$ for the above $\mathcal{G}$-game based quantifiers as for the ones specified quite differently in Definition 2 of Section VII. As shown in [6], we have

$$
v_{\mathcal{M}}\left(\Pi_{m}^{k} x F(x)\right)=\left(\begin{array}{c}
k+m \\
k
\end{array}\right)\left(\operatorname{Prop}_{F}\right)^{k}\left(1-\operatorname{Prop}_{F}\right)^{m}
$$

also in the current setting, where $\Pi_{m}^{k}$ is given by the above $\mathcal{G}$ game rule $R_{\Pi_{m}^{k}}^{\mathcal{H}}$. In the light of Section VII we thus obtain the perhaps surprising result that neither the relatively complex structure of $\mathcal{G}$-game states nor simultaneous bets for and against several instances of the scope formula are really needed to provide a game based semantics for these quantifiers. As stated in Corollary 2 one may alternatively specify $\Pi_{m}^{k}$ by (multiple-move) rules that respect Hintikka's Principle and thus fit the $\mathcal{H}$-game format.
However we hasten to point out that one cannot always trade simultaneous bets in complex $\mathcal{G}$-game states for complex (multi-move) rules referring to simple $\mathcal{H}$-game states. Indeed, for example the so-called blind choice quantifiers $\mathrm{L}_{m}^{k}$ and $\mathrm{G}_{m}^{k}$ introduced in [6], cannot be defined in our $\mathcal{H}$-game based setting. This follows from Theorem 9.2.4 in [10], showing that connectives like Łukasiewicz implication or strong Łukasiewicz conjunction, that are not definable in logic $\mathrm{KZ}$ and thus also not by $\mathcal{H}$-game rules, are needed to reduce those quantifiers to the basic choice quantifier $\Pi$.

\section{CONCLUSION}

We have explored the realm of monadic semi-fuzzy quantifiers specified by Hintikka-style game rules that may involve uniformly random choice and combine several moves. 'Hintikka style', here, refers to what we called Hintikka's Principle: each game state is completely determined by a single instance of a subformula of the original formula together with the current distribution of roles between the two strategic players. It turned out that the expressive power of such rules is considerable. We have shown that a family of quantifiers, that were originally defined in the context of the much more general setting of Giles's game for Łukasiewicz logic can already be specified by game rules that obey Hintikka's Principle. In particular, the simultaneous bets for and against several instances of the scope formula featured by Giles-style rules can be traded for Hintikka-style multi-move rules.

As a topic for future work it remains to characterize the class of all truth functions for quantifiers that can be defined in this manner. Furthermore, we have restricted attention to formulas built up from crisp predicates, here. However, in principle, our game semantic setting amounts to a quantifier fuzzification mechanism (cf. [2]) that deserves to be investigated for its own sake.

\section{ACKNOWLEDGMENT}

This work has been supported by FWF grants P25417-G15 (LOGFRADIG) and I1897-N25 (MoVaQ-MFL). We thank Paoli Baldi and Christoph Roschger for valuable comments on an earlier version of this paper.

\section{REFERENCES}

[1] L. Zadeh, "A computational approach to fuzzy quantifiers in natural languages," Computers \& Mathematics with Applications, vol. 9, no. 1, pp. 149-184, 1983.

[2] I. Glöckner, Fuzzy quantifiers: A computational theory, ser. Studies in Fuzziness and Soft Computing. Springer Verlag, 2006, vol. 193.

[3] M. Delgado, M. Ruiz, D. Sánchez, and M. Vila, "Fuzzy quantification: a state of the art," Fuzzy Sets and Systems, vol. 242, pp. 1-30, 2014.

[4] P. Cintula, C. G. Fermüller, P. Hájek, and C. Noguera, Eds., Handbook of Mathematical Fuzzy Logic (in three volumes). College Publications, 2011 and 2015.

[5] C. Fermüller and C. Roschger, "Randomized game semantics for semifuzzy quantifiers," in Advances in Computational Intelligence, ser. Communications in Computer and Information Science, S. e. Greco, Ed., vol. 300. Springer, 2012, pp. 632-641.

[6] - "Randomized game semantics for semi-fuzzy quantifiers," Logic Journal of the IGPL, vol. 22, no. 3, pp. 413-439, 2014.

[7] R. Giles, "A non-classical logic for physics," Studia Logica, vol. 33, no. 4, pp. 397-415, 1974. 
[8] J. Hintikka, "Language-games for quantifiers," in Studies in logical theory, N. Rescher, Ed. Blackwell, Oxford, 1968, pp. 46-72.

[9] P. Cintula and O. Majer, "Towards evaluation games for fuzzy logics," in Games: Unifying Logic, Language, and Philosophy, O. Majer, A.-V. Pietarinen, and T. Tulenheimo, Eds. Springer, 2009, pp. 117-138.

[10] C. G. Fermüller, "Semantic games for fuzzy logics," in Handbook of Mathematical Fuzzy Logic - Volume 3, P. Cintula, C. G. Fermüller, and C. Noguera, Eds. College Publications, 2015, pp. 969-1028.

[11] C. G. Fermüller, "Hintikka-style semantic games for fuzzy logics," in Proceedings of FoIKS 2014, ser. LNCS, C. Beierle and C. Meghini, Eds., vol. 8367. Springer, 2014, pp. 193-210.

[12] J. Hintikka and G. Sandu, "Game-theoretical semantics," in Handbook of Logic and Language, J. van Benthem and A. ter Meulen, Eds. Elsevier, 2010. 